\title{
Das Wurzener Kollegiat- und evangelisch-lutherische Domstift St. Marien
}

\author{
Alexander Wieckowski
}

Wurzen besitzt nicht nur einen eindrucksvollen mittelalterlichen Dom ${ }^{1}$ sondern auch ein Domkapitel, das auf eine durchgehende 900-jährige Tradition zurückblicken kann. ${ }^{2}$ Das evangelische Domstift St. Marien in Wurzen ist das einzige evangelische Stift im deutschsprachigen Raum, das auf ein mittelalterliches Kollegiatstift zurückgeht.

\section{Der historische Rahmen}

Unter einem Kollegiatstift versteht man eine rechtlich definierte Gemeinschaft von Weltgeistlichen, den sogenannten Kanonikern oder Chorherren, die in einem Stiftskapitel organisiert und durch die Stiftspfründen alimentiert waren. ${ }^{3}$ Ihr vorrangiger Einsatz galt dem gemeinschaftlichen Vollzug der Liturgie in den Stundengebeten und Gottesdiensten. Im Gegensatz zu den Mönchen unterstanden die Kanoniker dabei keiner Ordensregel und

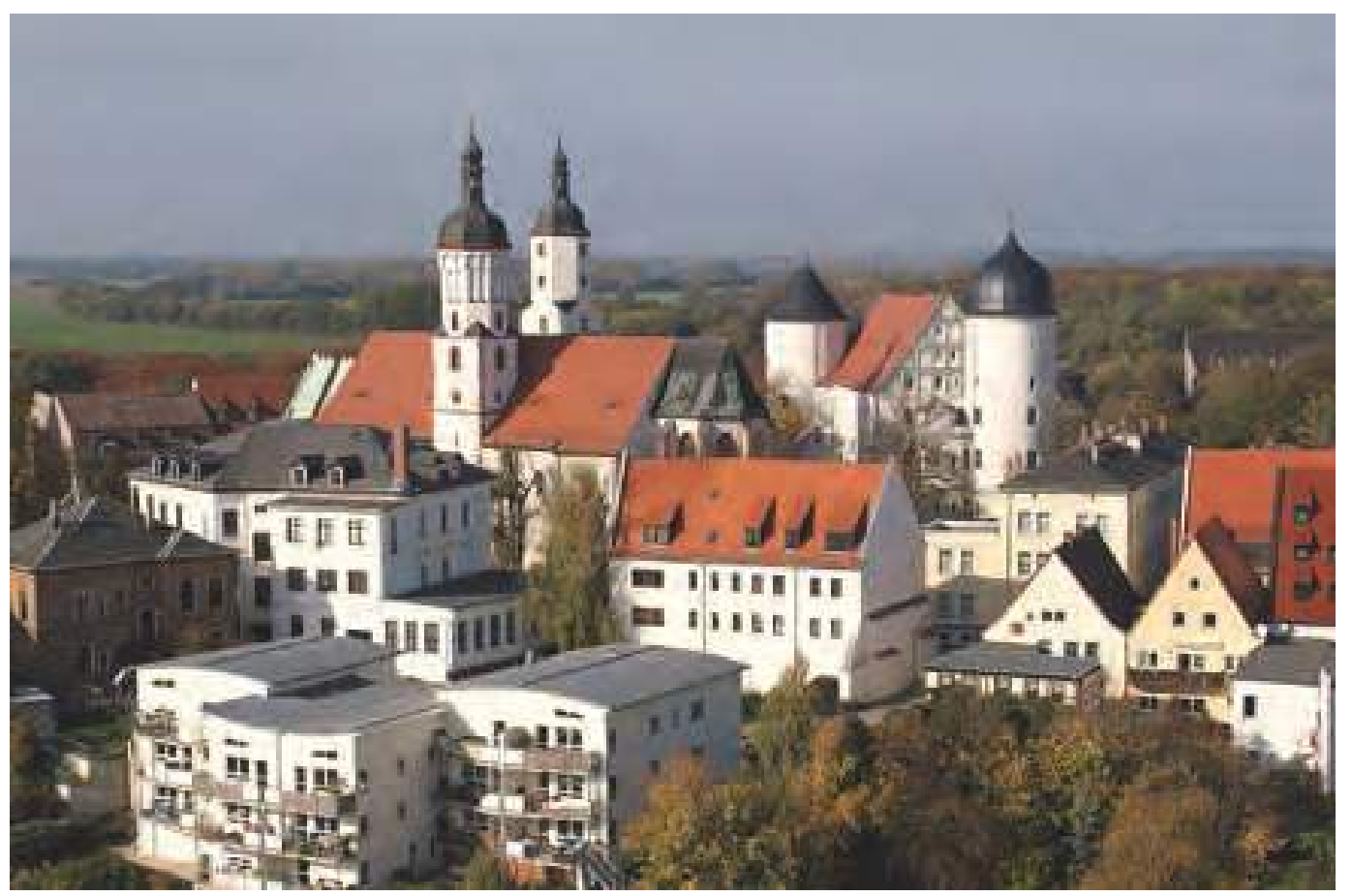

waren so auch nicht den Gelübden von Armut, Keuschheit und Gehorsam verpflichtet. Sie durften daher Besitz haben, waren erbberechtigt und testierfähig. Kollegiatstifte unterschieden sich von Domstiften weder in der Lebensform noch in der Verfassung, sondern allein im Hinblick auf das Recht der Bischofswahl, das nur den Domkapiteln zustand. So wurde der mittelhochdeutsche Begriff „tuom/thum“ mit seinen Erweiterungen „Thumkirche“ und „Tuomherren“ sowohl für Domkirchen und Domkapitel als auch für Kollegiatstifter und Kollegiatkapitel verwendet. ${ }^{4}$ Um dennoch den Unterschied des Wurzener Kollegiatstifts gegenüber dem Meißner Domstift zu verdeutlichen, verfügte Kurfürst Friedrich August III. als Stiftsherr 1770, dass der Gebrauch der Bezeichnung „Dom“ und folglich „Domstift“, „Domherren“ und „Domkapitel“ für Wurzen zu unterlassen sei. Aber seit 1929 trägt das Wurzener Kollegiatstift
1 Zum Dom vgl. Andrea Sander: Dom St. Marien zu Wurzen. Beucha/Markkleeberg 2014

2 Zum Wurzener Kollegiatstift im Folgenden vgl. Christian Schöttgen: Historie der Chur-Sächsischen Stifts-Stadt Wurtzen Leipzig 1717. Walter Schlesinger: Kirchengeschichte Sachsens im Mittelalter. Bd. 2: Das Zeitalter der deutschen Ostsiedlung (1100-1300). Köln/Wien 1962 2. Aufl. 1983, S. 203-205; Franz Lau: Aus der Geschichte des Domes und des Domkapitels zu Wurzen, in: Herbergen der Christenheit 3 (1961), S. 8-19; Ralf Thomas: Das Kollegiatstift St. Marien in Wurzen, in: Stiftsland Wurzen. Sächsische Kirchenverfassung. Historische Kirchenkunde: Aufsätze zur sächsischen Kirchengeschichte; hrsg. von Michael Beyer und Alexander Wieckowski. Leipzig 2011, S. 124-138. Zum mittelalterlichen Domstift zukünftig vor allem: Enno Bünz; u.a.: Wurzen, Kollegiatstift St. Marien, in: Sächsisches Klosterbuch. Die mittelalterlichen Klöster, Stifte und Kommenden im Gebiet des Freistaates Sachsen, Bd. 2: Marienstern - Zwickau, hrsg. von Enno Bünz in Zusammenarbeit mit Sabine Zinsmeyer und Dirk Martin Mütze [in Vorbereitung]. Als Überblicksdarstellung ist im Sax-Verlag Beucha-Markkleeberg in Vorbereitung: Alexander Wieckowski: Das Kollegiatstift St. Marien - das evangelische Domkapitel Wurzen.

Dom St. Marien und Bischofsschloss in Wurzen Foto: Wolfgang Ebert 
3 Einen guten Überblick über die sächsischen Kollegiatstifte bieten: Matthias Donath: Kollegiatkapitel und Kollegiatstifter im Bistum Meißen. Ecclesia Misnensis: Jahrbuch des DombauVereins Meißen 2002. Meißen 2002, S. 14-25; Enno Bünz: Die Dom- und Kollegiatstifte in den Bistümern Meißen, Merseburg und Naumburg - geographisch, chronologisch und typologisch betrachtet, in: Regular- und Säkularkanonikerstifte in Mitteldeutschland, hrsg. von Dirk Martin Mütze. Dresden 2011, S. 143-178.

4 Vgl. Enno Bünz: Das Domstift St. Petri zu Bautzen und seine Bücher. Eine Einführung, in: Katalog der Handschriften der Domstiftbibliothek Bautzen, bearb. von Ulrike Spyra und Birgit Mitzscherlich. Leipzig 2012, S. 12.

5 Hermann Kinne: Das Kollegiatstift St. Petri zu Bautzen von der Gründung bis 1569. Berlin/ Boston 2014

6 Matthias Donath: Das Kollegiatstift St. Georg in Zscheila, in: Ecclesia Misnensis: Jahrbuch des Dombau-Vereins Meißen 2002, S. 52-59.

7 Karl-Hermann Kandler: Das Kollegiatstift Freiberg und sein Verhältnis zum Hochstift Meißen, in: Monumenta Misnensia 7 (2005/06), S. 96-104.

8 Zur Stiftungsurkunde vgl. Leo Bönhoff: Die Stiftungsurkunde des Wurzener Kollegiatstiftes, in: Beiträge zur sächsischen Kirchengeschichte 27 (1913), S. 1-15; zur Diskussion um ihre Echtheit vgl. Thomas Ludwig: Die Urkunde Bischof Gerungs von Meißen vom 22. November 1154, in: Ostsiedlung und Landesausbau in Sachsen. Die Kührener Urkunde von 1154 und ihr historisches Umfeld, hrsg. von Enno Bünz. Leipzig 2008, S. $267 \mathrm{f}$.

$9 \mathrm{Zu}$ Wurzen vgl. die einzelnen Aufsätze von Thomas (siehe Anm. 2).

Kollegiatstift St. Marien in Wurzen, Kapitelsiegel von 1384 nun auch ganz offiziell die Bezeichnung „Domstift St. Marien“.

Kollegiatstifte gehörten neben den Pfarrkirchen, Klöstern und Kommenden zur kirchlichen Landschaft im Mittelalter. Ihre große Gründungswelle fiel in das 10. und 11. Jahrhundert. In dieser Zeit bestand im 968 gegründeten Bistum Meißen nur das Domkapitel am Bischofssitz in Meißen als geistliche Institution. Erst anderthalb Jahrhunderte später wurde $1114 \mathrm{mit}$ dem Wurzener Kollegiatstift eine zweite geistliche Gemeinschaft ins Leben gerufen.

Wurzen war das erste von fünf Kollegiatstiften in der Meißner Diözese. Im Zuge des Landesausbaus ab der zweiten Hälfte des 12. Jahrhunderts gründeten die Meißner Bischöfe weitere Kollegiatstifter, so St. Petri in Bautzen $(1213 / 18)^{5}$ und St. Georg in GroßenhainZscheila $(1226)^{6}$. In die zweite Gründungswelle von Stiftskirchen im Spätmittelalter gehörten das nur kurzzeitig bestehende bischöfliche Residenzstift St. Erasmus in Stolpen (1409/10) und das Stadtstift St. Marien in Freiberg $(1480)^{7}$. Weitere geplante Stiftsgründungen in Colditz (1373) und in Dresden (1400) kamen nicht zur Ausführung. Im Zuge der Reformation wurden schließlich fast alle Stifte aufgelöst, nur St. Petri in Bautzen blieb römisch-katholisch erhalten, und St. Marien in Wurzen wurde in das bis heute bestehende evangelische Domstift umgewandelt.

\section{Das mittelalterliche Kollegiatstift}

Im Jahr 1114 gründete Bischof Herwig (11061118 ) in Wurzen das Kollegiatstift. Die darüber ausgestellte Urkunde liegt nicht mehr im Original, sondern nur als Abschrift im „Liber Theodorici“, einem nach dem Meißner Bischof Dietrich III. von Schönberg benannten Kopialbuch, vor. ${ }^{8}$ Sie besagt, dass Bischof Herwig ein kleines Münster im Wurzener Land zu Ehren der heiligen Gottesgebärerin Maria errichtet und zusammen mit dem Naumburger Bischof Theodericus I. (1111-1123) geweiht habe. Das „Wurzener Land“ umfasste die Burgwarde Wurzen und Püchau, die zwischen 995 und 1040 in den Besitz des Meißner Hochstifts gekommen waren und in denen der Bischof Landesherr war. ${ }^{9}$ Dazu zählte auch die Exklave um Löbnitz und Pouch an der unteren Mulde. Strategisch günstig erhob sich in Wurzen, 961 erstmals erwähnt, der ottonische Burgward über das Muldental. Zwei wichtige Handelsstraßen kreuzten sich an der Furt der Mulde, die seit 1017 Grenze zwischen den Bistümern Meißen und Merseburg war. Mit dem Kollegiatstift in Wurzen schuf Bischof Herwig ein geistliches Zentrum in den neu aufgesiedelten Gebieten an der Westgrenze seines Bistums. Zugleich stärkte er im Wurzener Stiftsgebiet seinen Einfluss als bischöflicher Landesherr, den er in Meißen gegenüber den Markgrafen und Burggrafen gar nicht erst erlangen konnte. Die am 16. August 1114 geweihte Marienkirche wurde der geistliche Mittelpunkt des Stiftskapitels und erfuhr im Laufe der Jahrhunderte vielfache Erweiterungen und Umbauten. Zur materiellen Ausstattung des Stifts gehörte der Burgward Pouch bei Bitterfeld mit Kirche und Zehnten, der Wurzener Zoll, drei Dörfer in der Umgebung von Wurzen: Mischwitz, Trauschkau und Wurthau, die im Spätmittelalter wüst gefallen sind, ebenso neun Hufen in den Saaleorten Treben und Kleinkorbetha sowie zwei Lehnsgüter, deren Lage unbekannt ist. In Wurzen selbst stattete Bischof Herwig seine Gründung neben einem nicht weiter beschriebenen Lehen des erbenlosen bischöflichen Amtmannes Hermann nur noch mit dem Stiftsbezirk südlich der Domkirche aus, der 1358 durch Wall und Graben befestigt und somit von der Stadt getrennt wurde. Im Vergleich $\mathrm{zu}$ anderen Kollegiatstiftern war dies ein eher bescheidener Besitz an Gütern und Einkünften. Der größte Teil des Wurzener Stiftslandes blieb in bischöflicher Hand, gehörte also weiterhin dem Meißner Hochstift und kam nicht in den Besitz des Wurzener Kollegiatstifts. So gehörte der nördliche Teil des Domberges mit dem späteren spätgotischen Schloss zum bischöflichen Bereich und bildete zwischen 1559 und 1581 sogar die wichtigste Residenz des Meißner Bischofs.

Die knapp bemessene Erstausstattung des Kollegiatstiftes und die Bezeichnung der Kanoniker als „fratres“ (Brüder) in der Stiftungsur-

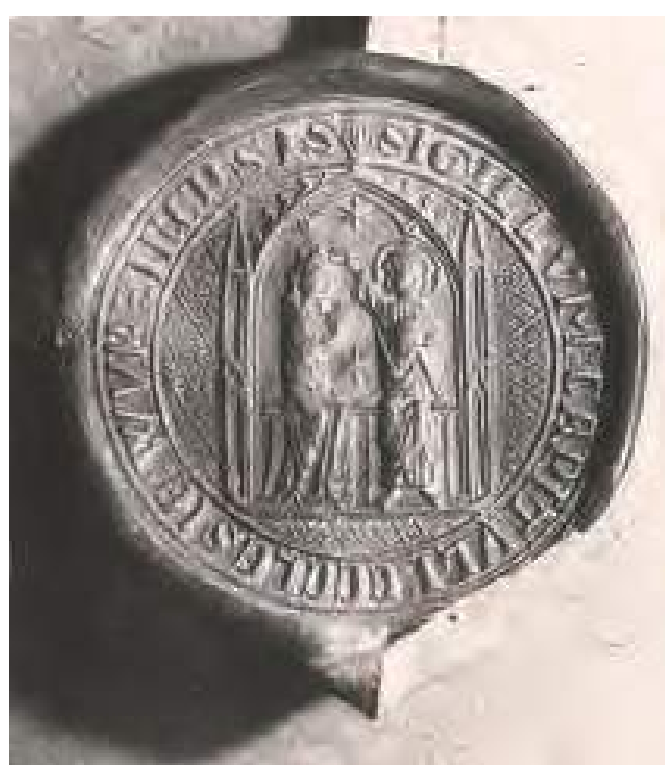


kunde lassen auf ein Gemeinschaftsleben der Stiftsherren nach der Aachener Regel schließen. Aber schon zu Beginn des 13. Jahrhunderts wird es in Wurzen wie auch anderswo zur Auflösung dieser vita communis gekommen sein. Man teilte den Stiftsbesitz in einzelne Pfründen (Präbenden) auf, gab sich ein eigenes Statut, das den Stiftsalltag regelte und baute Häuser, sogenannte Kurien, in denen die Kanoniker mit eigenem Haushalt lebten.

Die Anzahl der Stiftsherren war nicht festgelegt. ${ }^{10}$ Bei den Ansiedlungsverträgen von Kühren $1154^{11}$ und von Löbnitz 1185 unterzeichneten neben dem Propst drei weitere Kanoniker. 1223 werden sechs Stiftsherren genannt. Bis 1470 erhöhte sich die Anzahl auf elf Kanoniker, die bis zur Einführung der Reformation auch Bestand hatte.

Innerhalb des Kollegiatkapitels gab es unterschiedliche Rangstufen. An der Spitze des Kollegiatstifts stand der Propst, der zwar vom Kapitel gewählt wurde, aber bis auf eine kurze Unterbrechung im letzten Viertel des 15. Jahrhunderts immer aus den Reihen der Meißner Domherren entstammen musste. Im Laufe des Mittelalters wurde die Dignität des Propstes mit der Funktion eines Archidiakons verbunden. Der Propst übernahm in seinem Wurzener Bezirk, der sich östlich der Mulde von Jeßnitz bis Geringswalde erstreckte und 73 Pfarreien umfasste, bischöfliche Aufgaben, indem er den Klerus beaufsichtigte und die geistliche Gerichtsbarkeit ausübte.

Da der Propst als Meißner Domherr und als wichtiger bischöflicher Mitarbeiter nur selten in Wurzen residierte, übernahm der Dekan oder Dechant, erstmals 1223 erwähnt, die Stiftsleitung. Er verwaltete als Geschäftsführer die inneren Angelegenheiten des Kapitels, führte die offizielle Korrespondenz und übte die Disziplinargewalt über das Stift und seine Angestellten aus. Gewählt wurde der Dekan aus den Reihen der Kapitularen und war zur Residenz in Wurzen verpflichtet.

Neben diesen beiden Dignitäten gab es weitere Ämter. So werden erstmals 1211 der Scholaster, der Leiter der Stiftsschule, und 1283 der Kustos, zuständig für den Kirchenschatz und den laufenden Stiftskirchenbetrieb, genannt. Einen eigenen Kantor hat es im Wurzener Kapitel nicht gegeben.

Die Stiftsherren trafen sich regelmäßig freitags zu Kapitelsitzungen, deren Leitung der Dekan innehatte. Weiterhin gab es jährlich zwei Generalkapitel, und zwar montags nach dem Sonntag Kantate und nach dem Feiertag Mariae Geburt. Als Sitzungssaal diente wahrscheinlich die alte Domsakristei und ab 1515 ein großer Raum im neuerbauten Kapitelhaus. Die Stiftsherren legten sich ein eigenes Statut zu. Im Domarchiv hat sich ein Exemplar von 1476 erhalten, das ältere Überlieferungen integrierte sowie 1494 und 1505 Erweiterungen fand. ${ }^{12}$ Dieses Stiftsstatut regelte die Wahl und Annahme der Kanoniker, die Führung der Anwärterliste, die Pfründenverleihung, das Stimmrecht im Kapitel, die Einnahme des Chorstuhls, die Eidesformeln der Dignitäten, Kanoniker, Vikare und des Prokurators. Weiterhin enthält es neben Festlegungen zu den liturgischen Stundengebeten und Gottesdiensten auch Bestimmungen zum Betrieb der Stiftsschule, zu den Aufgaben des Schülerchors und zur Rechnungslegung durch den Prokurator.

$\mathrm{Zu}$ Herkunft und Ausbildung der Kanoniker gab das Statut keine Vorgaben. So setzte sich das Wurzener Kapitel aus Angehörigen niederadliger und bürgerlicher Familien zusammen. Wie bei anderen Kollegiatstiften auch, war es also gemischtständisch. Allein bei den Inhabern der Propstei fanden sich mit den Burggrafen von Meißen oder Leisnig auch Vertreter des hohen Adels. Nicht alle Kanoniker werden über eine umfassende Bildung verfügt haben. Aus einer Urkunde von 1358 kann entnommen werden, dass sich unter den fünf schreibunkundigen Meißner Domherren auch der Wurzener Propst befand. ${ }^{13}$ Dagegen treten seit Ende des 14. Jahrhunderts studierte und promovierte Kanoniker in Erscheinung.

Als eigenständige Körperschaft führte das Stiftskapitel ein eigenes Siegel. Das älteste Kapitelsiegel ist an einer undatierten Urkunde aus dem 1. Drittel des 13. Jahrhunderts angebracht und zeigt eine weibliche Figur mit Fahne und Kelch. Dagegen erscheint das bis heute benutzte Motiv der Verkündigung Marias zum ersten Mal an zwei Siegelurkunden von 1383 und $1384 .^{14}$

$\mathrm{Zu}$ den wichtigsten Merkmalen eines Stiftsherren zählte neben dem Sitz und der Stimme im Kapitel der Pfründenbesitz als persönliches Einkommen. ${ }^{15}$ Die Pfründen bzw. Präbenden umfassten finanzielle und materielle Leistungen und Rechte aus den Besitzungen des Kollegiatstiftes. Diese waren nicht vererbbar und wurden in einer bestimmten Reihenfolge innerhalb des Kapitels weitergegeben. Das Wurzener Stift war unter anderem in folgenden Orten - zum Teil auch nur zeitweise begütert: Bennewitz, Canitz, Crostigal, Gerendorf, Gossa, Kleinzschepa, Körlitz, Lautzschen, Leckau, Lossa, Lüptitz, Moschitz, Nedewitz, Nischwitz, Pouch, Schalitz, Schmerz, Sellnitz, Siedewitz, Wurthau und Zischwitz. Viele dieser

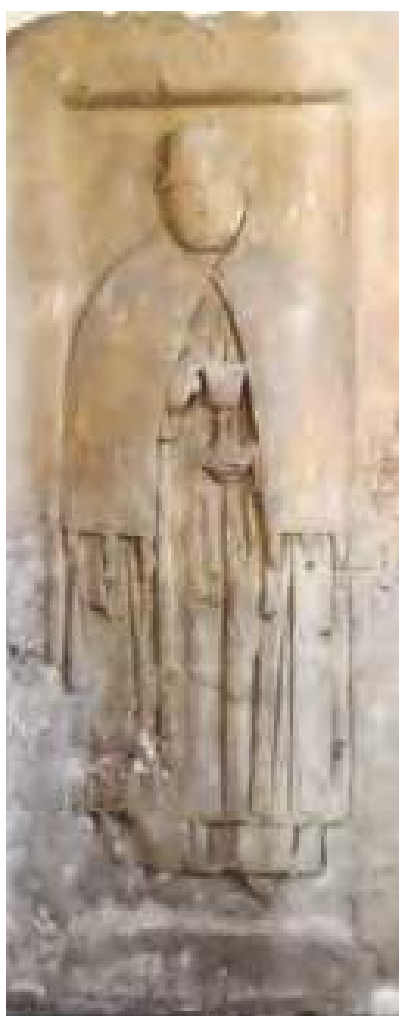

Dom St. Marien in Wurzen, Grabplatte eines Kanonikers, 15. Jahrhundert. Die Inschrift ist nicht mehr lesbar.

10 Zum Stiftskapitel vgl. Leo Bönhoff: Würdenträger und Mitglieder des Wurzener Stiftskapitels bis zur Kapitulation (10 Okt. 1581), in: Mitteilungen des Wurzener Geschichts- und Altertumsvereins 2, Heft 2 (1916), S. 48-76; Nachträge zu dem $\mathrm{Ar}$ tikel „Würdenträger und Mitglieder des Wurzener Stiftskapitels bis zur Kapitulation“ (1581), in Mitteilungen des Wurzener Geschichts- und Altertumsvereins 3, Heft 2, Teil 1 (1926), S. 60-62.

11 Vgl. die Neuedition der Kührener Urkunde von 1154 durch Thomas Wittig (siehe Anm. 8), S. $485-487$.

12 Archiv des Domstifts St. Marien Wurzen: Nr. 692: Statuta Capituli Wurzinensis; Schöttgen (siehe Anm. 2), Anhang, S. 63-108

13 CDS II, 2, S. 15, Nr. 506. Bei dem Wurzener Propst handelt es sich um Gerhard von Wolftitz.

14 Sächsisches Staatsarchiv, Hauptstaatsarchiv Dresden, Depositum des Meißner Domkapitels, Urkunden 195 und 494. 
Wurzener Dom und Domfreiheit vor 1880. Die Stiftsherren und Stiftsbediensteten lebten in den Häusern an der Domfreiheit.

15 Vgl. Leo Bönhoff: Die Pfründen der Stiftskirche U. L. Frauen in Wurzen, in: Mitteilungen des Wurzener Geschichts- und Altertumsvereins 2, Heft 2 (1916), S. 1-19.

16 Vgl. Neue Sächsische Kirchengalerie: Die Parochie rechts der Mulde. Leipzig 1914, Sp. 19-22; Wolfgang Ebert: Wurzen und die Muldenaue, Ein Führer durch die Stadt, ihre Landschaft und Geschichte. Beucha/Markkleeberg 2010, S. 34-49.
Orte waren aber schon im späten Mittelalter wüst gefallen. Im Jahr 1340 zählte man acht Präbenden, wobei die Pfründe Pouch, Wurzener Zoll sowie die Doppelpfründe Lüptitz und Bennewitz zu den ältesten gehörten. 1285 kam eine siebente Präbende St. Simon und Juda dazu. Die achte, gut ausgestattete Pfründe bildete die Wurzener Pfarrkirche St. Wenzeslai, die allerdings 1340 aufgehoben wurde. Man inkorporierte sie in das Kollegiatstift und besserte so die Einkünfte aller Kanoniker auf. Durch weitere Zustiftungen konnte die Präbendenanzahl bis 1470 auf elf angehoben werden: 1340 Präbende St. Margarethen, vor 1362 Präbende St. Barbara, um 1463 Präbende St. Laurentii und 1470 Präbende St. Erasmi.

Die Stiftsherren genossen die Einkünfte ihrer Pfründe bei einem Minimum von Verpflichtungen und waren somit auch abkömmlich und mobil. Ihre gottesdienstlichen Aufgaben delegierten sie an Vikare (lateinisch vicarius = Vertreter), die sie aus ihrem Vermögen bezahlten. Durch finanzielle Anreize, das heißt durch hohe Präsenzgelder, suchte man die Stiftsherren weiterhin zur Teilnahme an den liturgischen Feiern zu bewegen. Dennoch wurde das geistliche Leben im Spätmittelalter vor allem von den Vikaren gestaltet. So liest man im Stiftsstatut von einem Rector scholarum, der anstelle des Scholasters die Stiftsschule und den dazugehörigen Schülerchor leitete. Für die Verwaltung und Ordnung in der Stiftskirche sorgte neben dem Kustos hauptsächlich ein Subkustos. 1542 gab es in Wurzen zehn Vikarien. Diese besetzte man mit Messpriestern, den sogenannten Altaristen, die aber nicht dem Stiftskapitel angehörten. Eine ursprünglich elfte Vikarie wurde dem Predigtstuhl der Stiftskirche zugeschlagen. Obwohl die Wurzener
Stiftskirche keine Pfarrkirche war und die Stiftsherren und Vikare ihre Messen allein, also ohne Beteiligung von Gemeinde, feierten, gab es einen Stiftsprediger. Er war wie der Domprediger im Meißner Dom für die Familienangehörigen der Stifter von Seelenmessen, für die Pilger und Wallfahrer verantwortlich. Die Kleidung eines Domherrn bestand aus dem Superpelliceum, dem Chorrock, dem darüber getragenem Almutium, einem Pelzbehang, und dem Birett als Kopfbedeckung. Der einzige im Wurzener Dom erhaltene mittelalterliche Grabstein zeigt einen Kanoniker in einem solchen Ornat (siehe Abb. S. 221).

Das geistliche Zentrum des Kollegiatstiftes war über Jahrhunderte der südliche Teil der Domfreiheit. In unmittelbarer Nähe des Domes wohnten die Stiftsherren und Vikare in den Domherrenhäusern. Im Laufe der Jahrhunderte wurden viele Gebäude erneuert, verändert oder auch abgerissen. So mussten 1853 die Dechantei und die Scholasterei einem Schulbau weichen. Dem folgten die Abbrüche der Subkustodie 1891 und des Kapitelhauses mit dem Kreuzgang 1893. Erhalten geblieben sind die 1525 errichtete Kustodie, heute Domplatz 4, sowie die beiden sich anschließenden ehemaligen Domherrenkurien, Domplatz 5 und $6 .{ }^{16}$

Die Bedeutung des mittelalterlichen Kollegiatstiftes St. Marien ist in vier Punkten zu suchen. Zum einen stellte es ein geistliches Zentrum an der westlichen Meißner Bistumsgrenze dar, in dem der Gottesdienst und das liturgische Geschehen gefördert werden sollte. Dazu gehörte auch die Grabpflege und Memoria der dort beigesetzten Bischöfe Herwig und Johannes VI. von Salhausen (1487-1518). Ferner wurde das Kollegiatstift in die praktisch-administrative Erfassung des westlichen Teils der Meißner

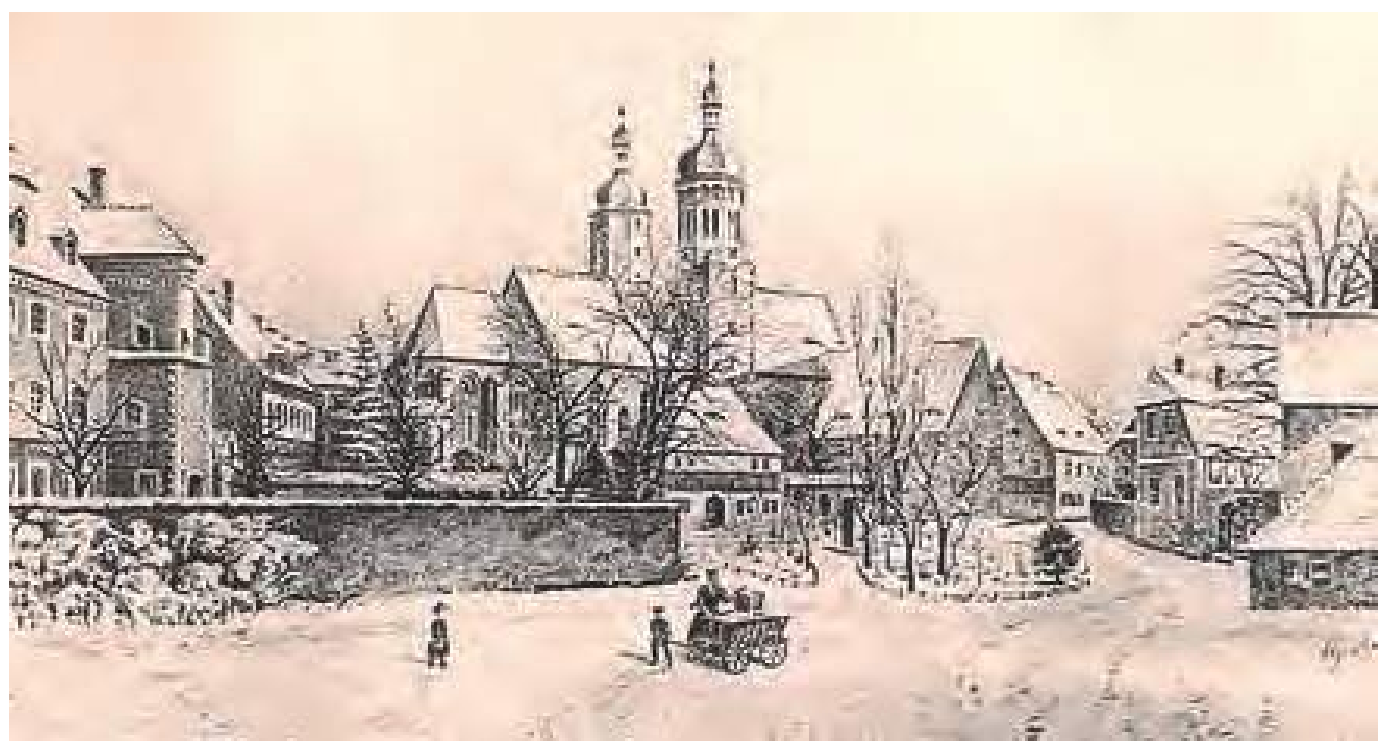


Diözese eingebunden. Dazu zählte das Patronat über die Pfarrkirche in Pouch, die Pfarrseelsorge in der Wurzener St. Wenzeslaikirche, der Unterrichtsbetrieb der Stiftsschule und die Mitwirkung des Propstes als Archidiakon in der Bistumsverwaltung. Zum anderen diente das Stift mit seiner Pfründenorganisation der Versorgung von Klerikern als Leistung- und Funktionsträgern. Sie standen als geistliche Räte und Richter oder als Kanzleibeamte, Notare und Prokuratoren in den Diensten der Bischöfe und weltlichen Landesherrn und waren somit eine wichtige Schnittstelle zwischen Kirche und Welt. Zudem stammen vier Meißner Bischöfe aus den Reihen der Wurzener Stiftsherren. Zuletzt war das Kollegiatstift für den Bischof auch ein entscheidender Machtfaktor in seinem Stiftsgebiet. Er sicherte sich seinen Einfluss durch die Kollatur von drei Präbenden sowie von Scholastikat und Kustodie.

\section{Das Kollegiatstift zur Reformationszeit}

Während der Reformationszeit konnte das Stift St. Marien innerhalb des hochstiftseigenen Wurzener Landes lange Zeit seinen Bestand erhalten. ${ }^{17}$ Mit der evangelischen Bewegung kam das Stift zum einen durch einzelne Domvikare in Kontakt, die in denjenigen Kirchgemeinden tätig waren, die sich der reformatorischen Lehre geöffnet hatten. Zum anderen setzte der Wurzener Stadtrat 1539 den Thammenhainer Pfarrer Johann Hoffmann zum evangelischen Prediger in der dem Stift zugehörigen Wenceslaikirche ein. Die Weichen für eine lutherische Reformation des Stifts wurden erst nach der friedlich beigelegten Wurzener Fehde, dem sogenannten Fladenkrieg, zu Ostern 1542 gestellt. Der Oschatzer Schlichtungsvertrag vom 10. April 1542 setzte schließlich die Reformation im Kollegiatstift durch. Noch am selben Tag nahm man drei Domherren in Gewahrsam; sie mussten Urfehde schwören. Weiterhin wurden die Nebenaltäre, einige Bilder und das Sakramentshaus beseitigt sowie die Grabanlage des Bischoffs Johann von Salhausen zerstört.

Einen Tag später, am 11. Mai 1542, begannen die Visitatoren mit ihrer Arbeit. Die Stiftskirche, der Wurzener Dom, wurde zur Wurzener Pfarr- und Hauptkirche. Anstelle des Stiftskapitels übernahm der ernestinische Kurfürst die Kollatur für die Pfarr- und die Diakonenstelle. Eine neu begründete dritte geistliche Stelle, später Subdiakonat genannt, wurde vom Stadtrat besetzt. Der bisherige evangelische Prediger an der Wenceslaikirche, Johann Hoffmann, wurde zum Pfarrer an der Dom- und Hauptkirche bestellt und zugleich zum Superintendenten für die vierzehn im Stiftsgebiet liegenden Kirchgemeinden ernannt. Die Visitatoren entbanden die Stiftsherren und Vikare von allen ihren gottesdienstlichen Verpflichtungen. Der Stiftsbesitz wurde inventarisiert, die Stiftsschule vereinigt mit der Stadtschule. Das Kollegiatstift musste so zum Unterhalt des neugeordneten Wurzener Kirchenwesens einschließlich seiner Kirchen- und Schuldiener beitragen. Zum Zeitpunkt der Visitation waren von den elf Kanonikern und zehn Vikaren nur jeweils fünf anwesend. Von ihnen bekannten sich zwei Domherren zur Reformation.

Durch den Verlust der Stolpener Bischofsresidenz 1559 verlegte der letzte Meißner Bischof und vorherige Wurzener Domdechant, Johann IX. von Haugwitz (1555-1581, gest. 1595), seinen Diözesansitz nach Wurzen. Der hiesige Dom wurde zur „Ersatzkathedrale“. Unter seinem Schutz konnte sich das Kollegiatkapitel behaupten. 1579 unterschrieb der Meißner Bischof die Konkordienformel und bekannte sich zum lutherischen Glauben. Damit war der Weg für die endgültige Einführung der Reformation im Kollegiatstift frei. Zwischen dem Stiftskapitel und dem Stadtrat von Wurzen kam es 1580 zur Unterzeichnung eines umfangreichen Vergleichs, der die Anerkennung der Augsburgischen Konfession und die Wahrung der Gerechtsame des Stiftskapitels beinhaltete und zugleich die Besetzung und Bezahlung der Kirchen- und Schuldiener regelte. So sollte das Pfarramt unter der Kollatur des Kapitels stehen. ${ }^{18}$

Die am 10. Oktober 1581 zwischen Kurfürst und Bischof ausgehandelte und zehn Tage später ratifizierte Kapitulation bedeutete das Ende des alten Meißner Bistums. Johannes von Haugwitz resignierte zugunsten des Meißner Domkapitels. Der Bischofsstuhl war damit vakant. Kurfürst August von Sachsen wurde zum Administrator des Bistums Meißen. Die Meißner Domherren bestimmten Kurfürst August zum neuen Stiftsherrn und wählten auch die nachfolgenden Kurfürsten zu Stiftsherren, die zugleich stets immer auch Stiftsherrn des Wurzener Kollegiatkapitels waren. Der 7. Artikel der Kapitulation sicherte dem Wurzener Kollegiatstift und seinen Kapitularen den Erhalt des gegenwärtigen Rechtszustandes zu, freilich nun in evangelisch-lutherischer Ausprägung. Seit 1663 war die Stiftsherrschaft erblich mit dem sächsischen Kur- und späteren Königshaus verbunden. An dieser Ordnung änderte auch der 1697 erfolgte Über-
17 Zum Folgenden vgl. Karl August Hugo Burkhardt: Geschichte der sächsischen Kirchen- und Schulvisitationen von 1524 bis 1545 . Leipzig 1879 , S. 208-210; Leo Bönhoff: Die Einführung der Reformation in Stift, Stadt und Land Wurzen, in: Mitteilungen des Wurzener Geschichts- und Altertumsvereins 3, Heft 1 (1918), S. 34-36; Alfred Schultze Die Rechtslage der evangelischen Stifter Meißen und Wurzen: zugleich ein Beitrag zur Reformationsgeschichte. Leipzig 1922, S. 7-10; Ralf Thomas: Die Einführung der Reformation im Meißner Stiftsgebiet unter besonderer Berücksichtigung des Wurzener und Mügelner Territoriums, in Stiftsland Wurzen (siehe Anm. 2), S. 107-110; ders.: Das Wurzener Land im Jahre 1581, in: Stiftsland Wurzen (siehe Anm. 2), S. 115-123.

18 Schöttgen (siehe Anm. 2), S. 129-136. 
tritt von Kurfürst Friedrich August I. (16701733) zum römisch-katholischen Bekenntnis nichts.

\section{Das Kollegiatstift unter wettinischer Stiftsherrschaft}

Für das Stiftsland des Hochstifts Meißen um Wurzen und Mügeln, das als eigenständiges Territorium bis 1818 erhalten blieb, wurde in Wurzen eine Stiftsregierung als weltliche Verwaltungsbehörde eingesetzt. Zudem diente das Stiftskonsistorium mit dem General- bzw. Stiftssuperintendenten als kirchliche Aufsichtsbehörde. Die Geschicke des Wurzener Stiftslandes bestimmte das Meißner Hochstift und nicht das Wurzener Kollegiatstift. Dafür tagte das Meißner Domkapitel wiederholt in Wurzen, vollzog hier Huldigungen und nutzte dabei auch den Wurzener Dom als Gottesdienststätte. Der Wurzener Stiftssuperintendent wurde vom Meißner Domkapitel bestimmt und feierlich eingeführt; die Wurzener Stiftsherren saßen dabei in ihrem Gotteshaus nur unter den geladenen Honoratioren. Das Wurzener Stiftsland gliederte sich in Stiftsstände. $\mathrm{Zu}$ diesen gehörte auch das Wurzener Domkapitel. Seit 1666 zählten die Stiftsstände zu den Landständen und waren somit als Körperschaften im sächsischen Landtag vertreten. Während das Meißner Domkapitel einen Sitz in der ersten Kurie der Prälaten und Herren bekam, gehörte der Vertreter des Wurzener Stifts zur zweiten Kurie der Ritterschaft. Auch in der sächsischen Verfassung von 1831 besaßen beide Stifter Sitz und Stimme in der Ständeversammlung. So wurde jeweils ein Domherr aus Meißen und Wurzen in die erste Kammer des sächsischen Parlaments abgeordnet. Auf diese Weise konnte man Einfluss auf die Landespolitik nehmen. ${ }^{19}$

Weitere Herausforderungen für das Wurzener Kollegiatkapitel bestanden in der Ausübung der Patronate sowie in der Aufsicht und Verwaltung ihres Stiftsbesitzes. Ein Platz im Kollegiatstift war weiterhin begehrt, denn er brachte beträchtliche finanzielle Einkünfte. Neben den sieben Stiftsherren mit Sitz und Stimme im Kapitel gab es mit den Präbendaten und Vikaren weitere Personengruppen, die verschiedene Einkünfte aus dem Stiftsvermögen erhielten. ${ }^{20}$ Für alle Stellen existierte eine Warteliste für Bewerber, die sogenannten Expektanten. Vielfach betrachtete man das Kollegiatstift als eine gute Versorgungseinrichtung. Die Stiftsherren hatten keine geistlichen Verpflichtungen mehr, das gottesdienstliche Leben im Dom gestaltete die Wurzener Kirchgemeinde. Gemeinschaftlich mit dem Wurzener Stadtrat übte man die Kollaturrechte über die beiden Wurzener Pfarrstellen, das Schulrektorat und das Kirchneramt aus. Weiterhin besaß man alleinige Kollaturrechte an dem Wurzener Organistenamt sowie an den Pfarr- und Schulstellen in Kühren und
Kapitelstube in der Kustodie, um 1938
19 Siehe Josef Matzerath: Aspekte sächsischer Landtagsgeschichte: Die Mitglieder der (kur-) sächsischen Landstände 17631831. Dresden 2009; ders.: Die Mitglieder und Wahlbezirke der sächsischen Landtage (18331952). Teil I: 1833-1918. Dresden 2011.

20 Vgl. die einzelnen Aufstellungen in Königl. Polnischer und Churfürstl. Sächsischer Hoffund Staatskalender - Sächsische Staatshandbücher.

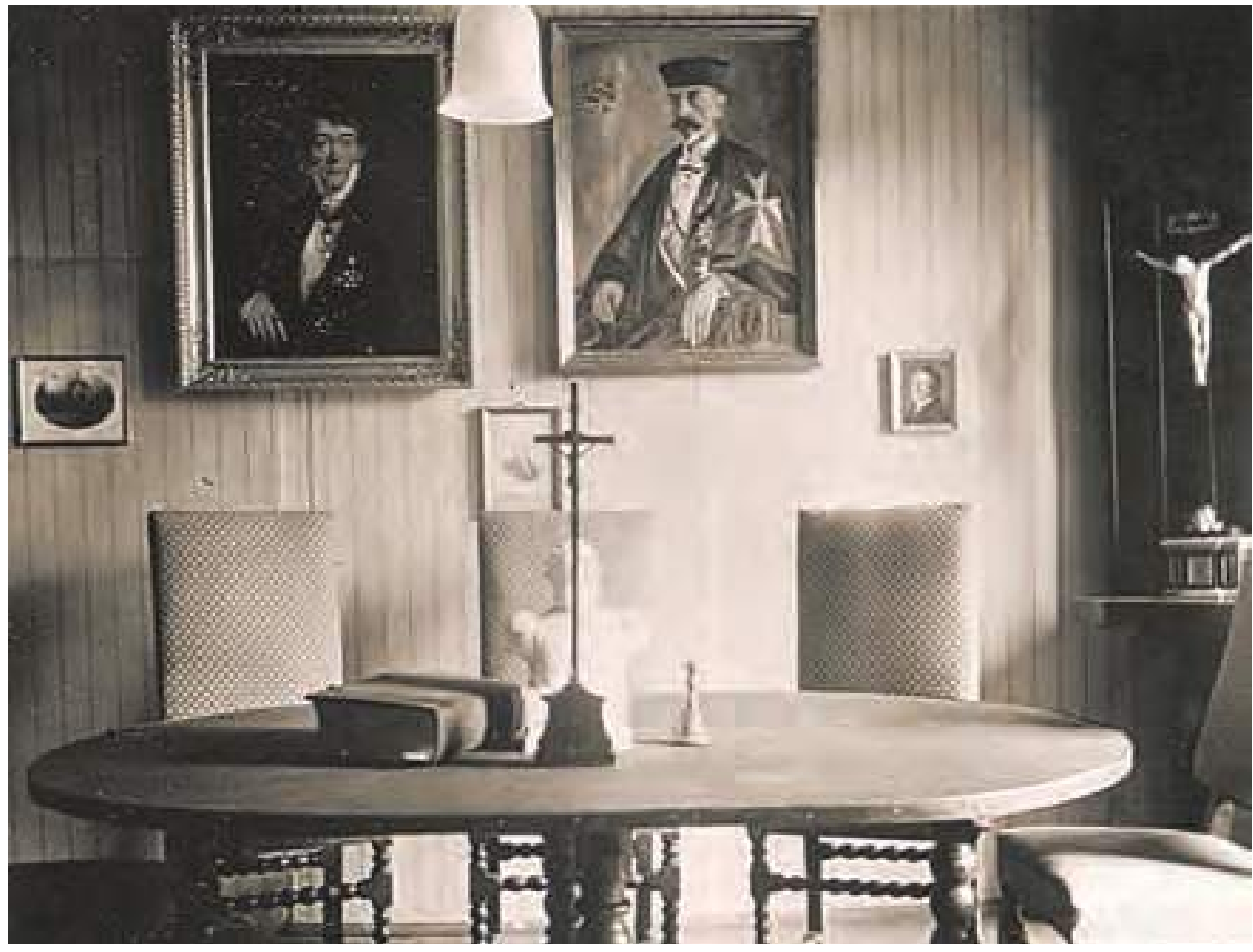


Lüptitz. ${ }^{21}$ Zudem vergaben die Domherren verschiedene stiftische Freistellen und Stipendien. Eine wesentliche Aufgabe des Stiftskapitels beinhaltete die Aufsicht der Vermögensrechte des Kollegiatstifts. Der Besitz speiste sich aus den Gütern und Einkünften der nach wie vor bestehenden Präbenden und Vikarien. Die Verwaltung der Stiftskasse oblag einem ausgewiesenen Juristen, der beim Stiftskapitel als Stiftssyndikus und Prokurator angestellt war. Ihm unterstand zudem die seit dem Mittelalter bestehende Dombaukasse, die sogenannte Fabrica. Aus ihr musste der Erhalt des Wurzener Doms finanziert werden.

Das 19. Jahrhundert mit seinen Umbrüchen, Reformen und Revolutionen ging auch nicht spurlos am Wurzener Kollegiatstift vorüber. Durch die Beschlüsse des Wiener Kongresses verlor das Stift seine Liegenschaften bei Pouch und Löbnitz an das Königreich Preußen. Die sächsische Agrarreform von 1832 beendete die grundherrschaftlichen Rechte des Stiftes. Die seit Jahrhunderten bestehenden Natural- und Zinsabgaben wurden in einmalige Geldzahlungen umgewandelt. So kam es zu zahlreichen Ablösungsverträgen zwischen dem Wurzener Kollegiatstift und dem Wurzener Rentamt, der Wurzener Fleischerinnung, den Besitzern der Wurzener Stadtfelder, den Besitzern der Tauchnitzmühle sowie der Mühle in Nemt und Kleinzschepa, weiterhin mit den Grundstücksbesitzern in Bortewitz, Burkartshain, Canitz, Collmen, Dehnitz, Körlitz, Kühren, Lüptitz, Mark Sellnitz, Nepperwitz, Roitzsch, Tallwitz und Watzschwitz sowie mit den Kirchlehen in Böhlitz und Treben. Aus den gezahlten Ablösegeldern wurde schließlich eine Kapitalstiftung errichtet, die im Jahr 1917 über 288.730 RM (im Vergleich das Domkapitel Meißen: 1.029.500 RM) verfügte. Die aber bald darauf folgende Inflation machte diesen Stiftungsfonds zunichte.22

Auch die Gerichtsbarkeit, die das Stiftskapitel wahrgenommen hatte, musste aufgegeben werden und wurde verstaatlicht. Eine teilweise und noch freiwillige Abtretung erfolgte Ende 1842. Am 2. Januar 1843 gingen die Jurisdiktionsbefugnisse über die Wurzener Kapitelgemeinde an das Königliche Landgericht Wurzen. Auf Anordnung des Justizministeriums erhielt dieses Gericht am 22. Dezember 1855 auch die noch verbliebene Gerichtsbarkeit des Stiftskapitels, die sich über 19 Häuser der Domfreiheit, über Lüptitz, die Sonnenmühle bei Oelschütz, Grundstücke in der Mark Lautzschen und über Wiesen in der Pausitzer Flur erstreckte. ${ }^{23}$

Von den einst vielfältigen Aufgaben sind den beiden Stiftern Wurzen und Meißen nicht

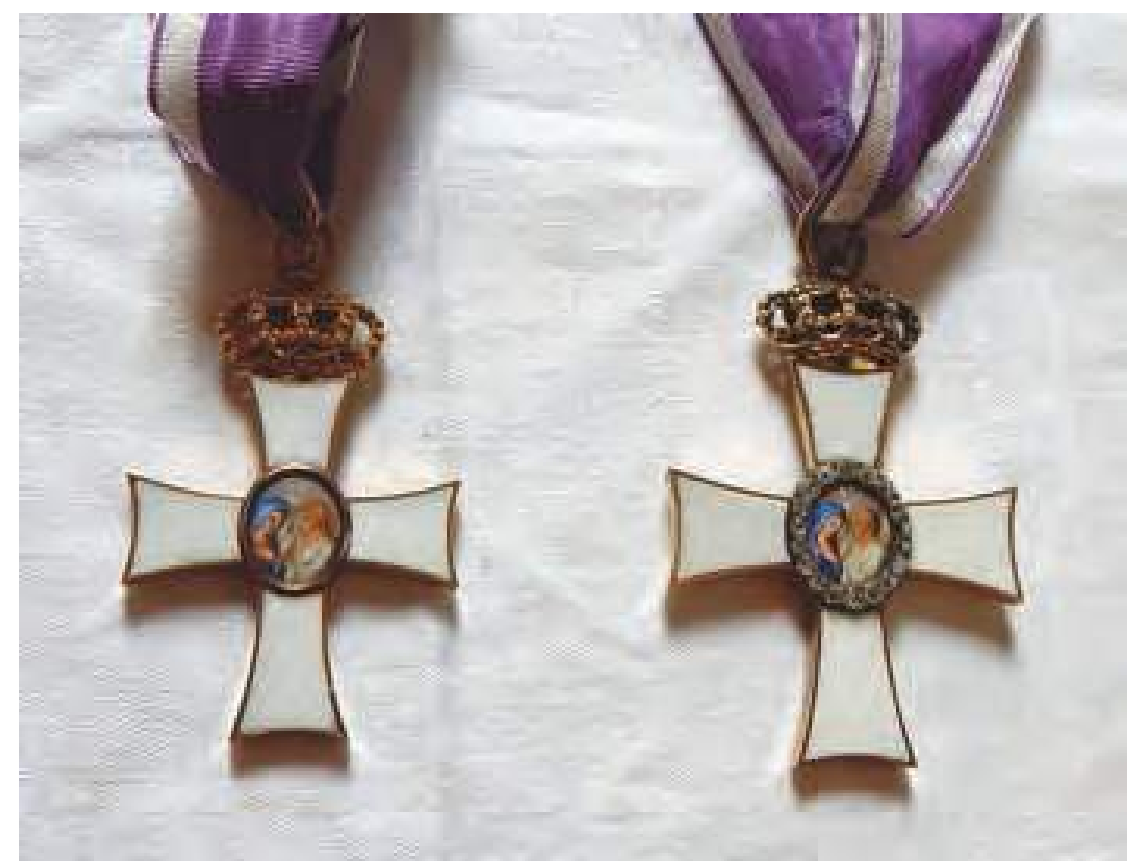

mehr viele geblieben. So wurden seit 1832 Stimmen laut, die nach einer Reform riefen. ${ }^{24}$ Die Stiftseinkünfte sollten für Kirchen- und Schulzwecke verwendet werden. Seit 1848 verhandelte man sogar über eine Aufhebung beider Kapitel. Der am 5. März 1851 von den Meißner und Wurzener Kapitelmitgliedern sowie dem Kultusministerium unterschriebene Auflösungsvertrag wurde aber nicht ratifiziert, vielmehr kam es 1859 für Meißen und 1864 für Wurzen zu Reformverträgen. Das Wurzener Kollegiatkapitel bestand weiterhin aus sieben Stiftsherren. Als sächsische Staatsbürger und lutherische Christen sollten sie ein Universitätsstudium absolviert und Verdienste in Kirche, Schule und Staat erworben haben. Eine wichtige Neuerung bestand in der Festsetzung der Bezüge. So erhielt der Propst 400 Taler, der Dechant 500, der Scholastikus 300, der Kustos 250, der fünfte Kapitular 200, der sechste 150 und der siebente 100 Taler. Im Jahr 1899 kam es zu einer Änderung des Reformvertrages. Die Zahl der Stiftsherren wurde auf fünf beschränkt und die Gesamtbezüge auf 6.400 Mark herabgesetzt.

Der Kapitular erhielt bei seiner Ernennung neben dem schwarzen Domherrenmantel, der auf der linken Brustseite ein weißes Kreuz zeigt, den Stiftsorden. Diesen stiftete 1808 König Friedrich August I. von Sachsen (17501827) in zwei verschiedenen Ausführungen, zum einen als Prälaturordenskreuz mit 18 Brillanten für den Dompropst, Dechanten und Senior und zum anderen als einfacheres Kanonikatsordenskreuz für die weiteren Kapitulare. In der Mitte des Kreuzes ist auf der Vorderseite
Stiftsorden des Kollegiatstifts St. Marien in Wurzen, gestiftet 1808 , links Kanonikatsordenskreuz, rechts Prälaturordenskreuz
21 Vgl. Schultze (siehe Anm. 17), S. 95.

22 Vgl. Schultze (siehe Anm. 17), S. 36 .

23 Sächsisches Staatsarchiv, Staatsarchiv Leipzig, 20077, Königliches Landgericht Wurzen, Amt Wurzen, Nr. 924, 956, 1007, 1009, 1014, 1054, 1246; 20580, Domkapitel Wurzen (Patrimonialgericht).

24 Zum Reformprozess und zu den Reformverträgen vgl. Gerhard Schmidt: Die Reform des Hochstifts Meißen im 19. Jahrhundert, in: Das Hochstift Meißen, hrsg. von Franz Lau. Berlin 1973, S. 301-322; Schultze (siehe Anm. 17), S. 19-36. 92-97. 
25 Archiv des Domstifts St. Marien Wurzen, Nr. 820.

26 Vgl. Schultze (siehe Anm. 17), S. 37-74.

27 Vgl. Horst Schulze: Das Domkapitel und die Hermann-IlgenStiftung als Auftraggeber bei der Neugestaltung des Domes St. Marien zu Wurzen, in: Georg Wrba (1872-1939): Im Schatten der Moderne, hrsg. von Thomas Pöpper. Leipzig 2009, S. 116-132. Der Beitrag findet sich ebenso in: Friedrich Hermann Ilgen. Eine Biographie, hrsg. vom Wurzener Geschichts- und Altstadt-Verein e.V. Wurzen 2013, S. 50-57.

28 Vgl. Dieter Auerbach: Verpflichtung und Gefährdung: Ereignisse aus der Geschichte des Domkapitels zu Meißen von den Anfängen bis zur friedlichen Revolution 1989, in: Monumenta Misnensia 8 (2007/08), S. 176-180.

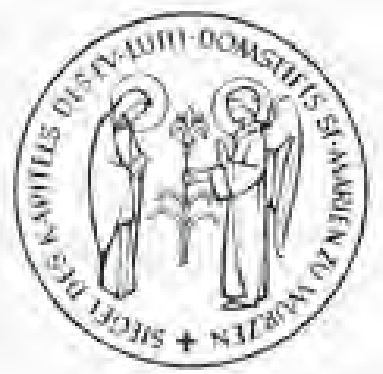

Domstift St. Marien, Kapitelsiegel, neue Fassung von 1963 die Darstellung der Verkündigung Mariae und auf der Rückseite der von Palmenzweigen umgebene Name des Königs zu sehen. ${ }^{25}$ Jeder Stiftsherr hatte im Dom seinen festen Platz. Vom mittelalterlichen Chorgestühl blieb nichts erhalten, vom neugotischen Domherrengestühl überdauerten einzelne mit rotem Samt bezogene Stühle. Das jetzige Domherrengestühl gehört zur Wrba-Bronzeausstattung von 1932 und befindet sich zu je vier Sitzen an der Nord- und Südwand des Domchors. An der Lehne sind die Wappen und Namen der 1932 amtierenden Kapitulare und des Stiftssyndikus festgehalten. die stiftsherrlichen Rechte vom jeweiligen Stiftsherrn des Meißner Hochstifts ausgeübt werden. Seitdem ist der jeweils amtierende sächsische Landesbischof zugleich Stiftsherr des Wurzener Domstifts. Die inneren Angelegenheiten regelte die neue Satzung von 1928. Die Anzahl der Mitglieder wurde wieder auf sieben angehoben.

Eine notwendige Domsanierung stand 1930 auf dem Plan. Einzelne Domherren forcierten dabei mit Hilfe der Hermann-Ilgen-Stiftung eine völlige Neugestaltung des Dominneren durch Georg Wrba. Die überaus starke Repräsentation des Domkapitels in der Gestaltung
Dom St. Marien in Wurzen, Domherrengestühl, 1932

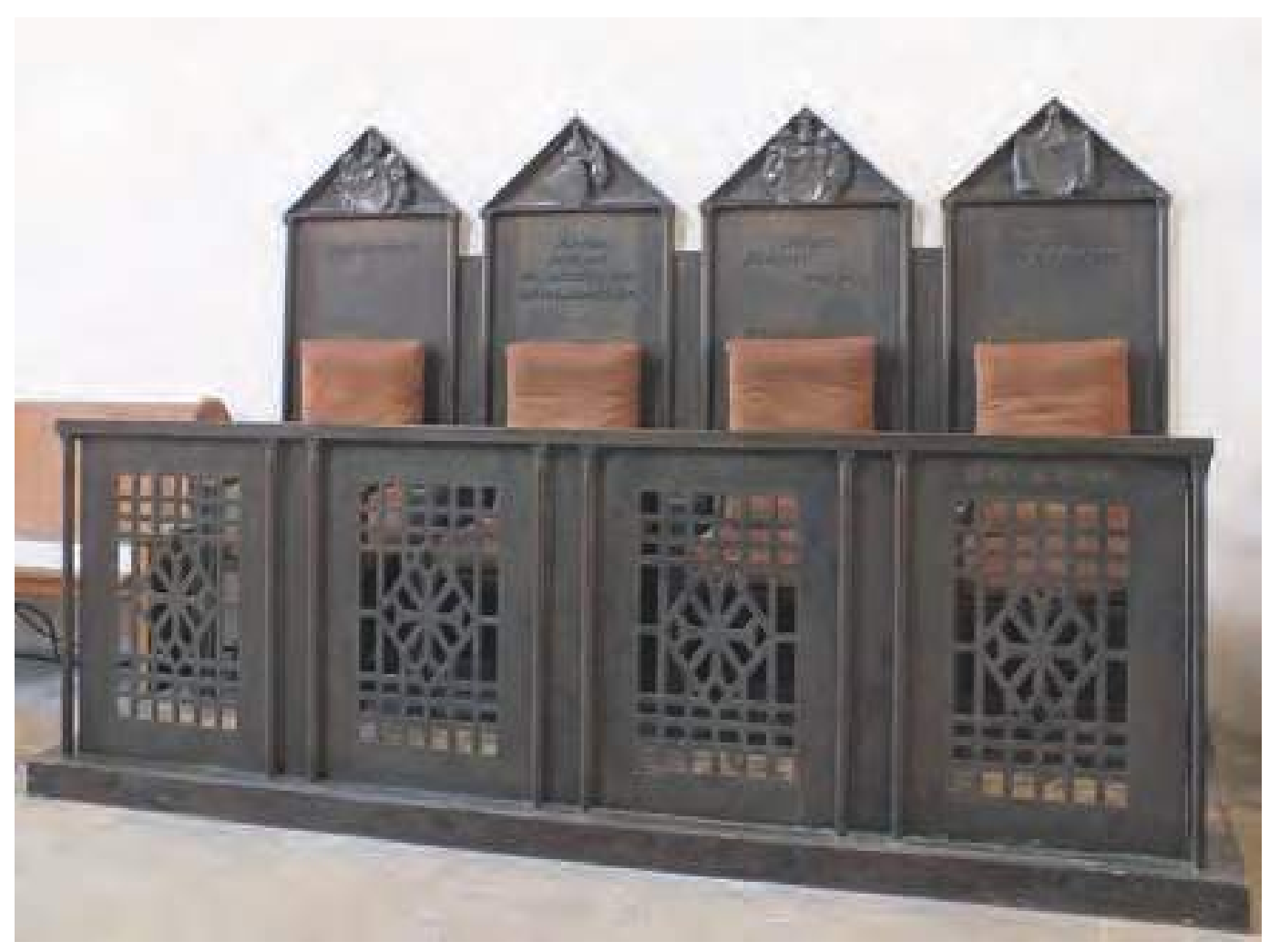

\section{Das Kollegiatstift im 20. und 21. Jahrhundert}

Das Ende der Monarchie 1918 empfanden viele Domherren als schmerzlichen Einschnitt. Mit der Abdankung von König Friedrich August III. (1865-1932) im November 1918 verloren beide Stifter ihren Stiftsherrn. Die neue Weimarer Reichsverfassung schrieb die Trennung von Staat und Kirche fest. Damit war eine Sedisvakanz eingetreten. 1924 wählten die Meißner Domherren Landesbischof Dr. Ludwig Ihmels (1858-1933) zu ihrem neuen Stiftsherrn. ${ }^{26}$ Das Kapitel des Domstifts Wurzen so nun die offizielle Bezeichnung - schloss mit der sächsischen Landeskirche 1925 ein Abkommen, in dem festgeschrieben wurde, dass des Domherrengestühls und besonders der Kanzel mag befremden, zeigen doch die Apostelköpfe an der Kanzel die porträthaften Züge aller Stiftsherren. ${ }^{27}$ Die Domweihe fand am 23. Oktober 1932 statt. Ein viertel Jahr später erfolgte die Machtergreifung der Nationalsozialisten im Reich, wenig später die Machtergreifung der Deutschen Christen in der sächsischen Landeskirche. Der neugewählte Bischof Friedrich Coch (1887-1945), ein überzeugtes NSDAP-Mitglied, lehnte das Amt des Stiftsherrn ab und so blieb dem Hochstift Meißen und dem Kollegiatstift Wurzen eine braune Stiftsherrschaft erspart. ${ }^{28}$

Erst 1947 wurde mit Bischof Hugo Hahn (1886-1957) ein neuer Stiftsherr berufen. Während des Zweiten Weltkriegs hatte die Ar- 


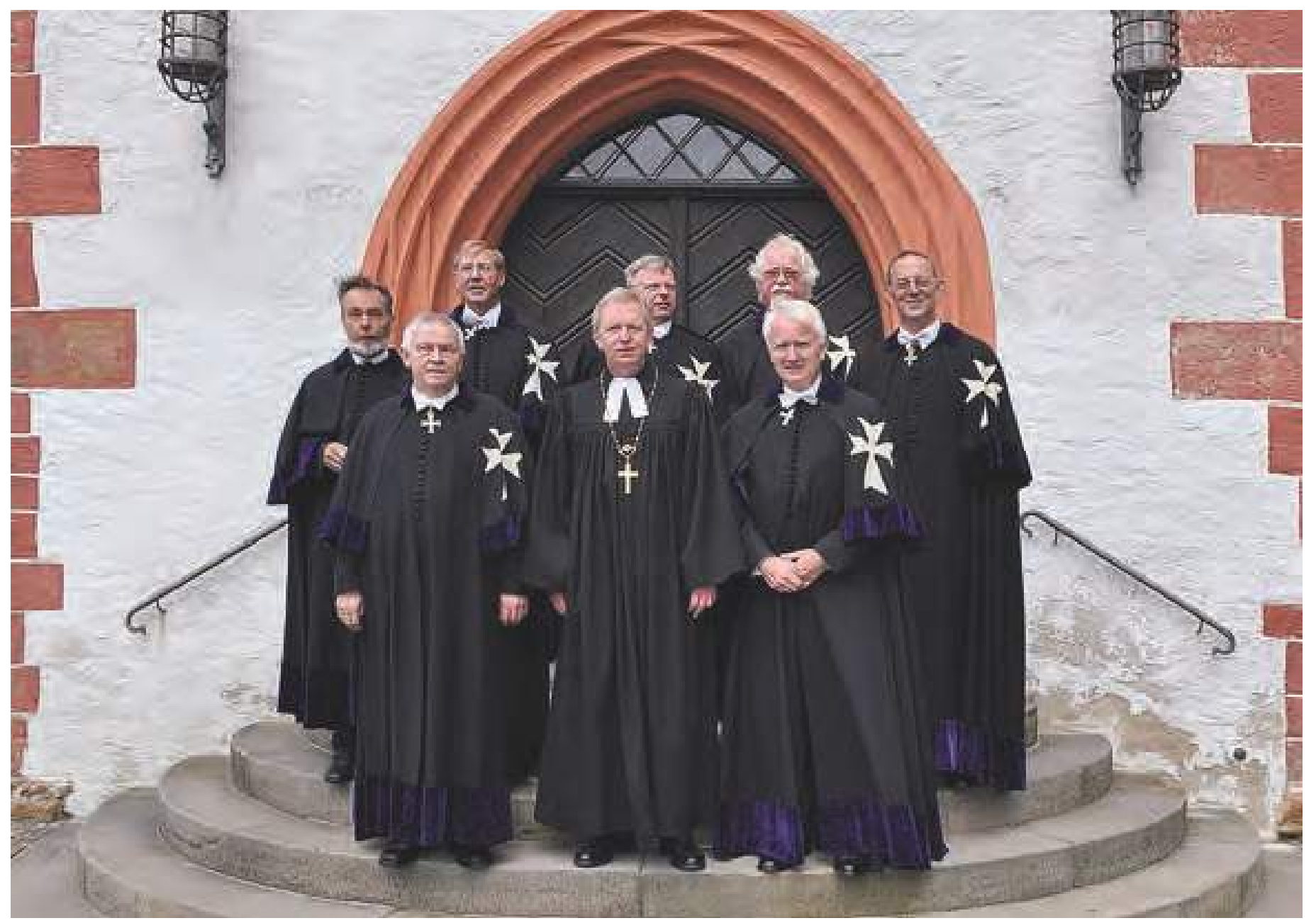

beit des Domkapitels geruht. Viele Domherren verstarben. Zu Kriegsende bestand das Kapitel aus nur noch zwei Kapitularen. Man strebte die Vereinigung der Kapitel von Meißen und Wurzen an, indem für beide dieselben Domherrn berufen wurden. Letztlich blieben die Stifter aber eigenständig.

Die aktuelle Satzung stammt von 1997. Ein Domherr nimmt seine Aufgabe im Ehrenamt mit allen Rechten und Pflichten nur bis zur Vollendung des 75. Lebensjahres wahr und kann danach mit beratender Stimme an den mehrmals im Jahr stattfindenden Konventen teilnehmen.

Die wichtigste Aufgabe des Domkapitels besteht in der Erhaltung des Doms. Seine in der DDR-Zeit immer schlechter werdende Bausubstanz und die staatliche Mangelwirtschaft führten zur Überlegung, den Dom aufzugeben bzw. an die katholische Kirche im Tausch mit der Herz-Jesu-Kirche abzugeben. Vor allem dem Wurzener Superintendenten und Domdechanten Horst Schulze ist es $\mathrm{zu}$ verdanken, dass der Dom seit 1980 schrittweise außen und innen erneuert werden konnte. ${ }^{29}$ Heute ist der Dom nicht nur die Heimstätte des Domkapi- tels und der Wenceslaikirchgemeinde, sondern auch das geistliche Zentrum der Stadt und die Festkirche des Kirchenbezirks Leipziger Land. Ein Höhepunkt ist der jeweils in den Sommermonaten stattfindende Domherrentag.

Zum derzeitigen Domkapitel ${ }^{30}$ gehören als Dompropst Superintendent i. R. Christoph Richter, als Dechant der Bankkaufmann und Jurist Leo Sahrer v. Sahr v. Schönberg, als Senior Kirchenbaurat Dr. h.c. Eberhard Burger und als Kapitulare Oberkirchenrat i. R. Dietrich Lenk, der emeritierte Mathematikprofessor Dr. Volker Nollau, der emeritierte Theologieprofessor Dr. Wolfgang Ratzmann und der Wurzener Domkantor und Organist Johannes Dickert. Zu den Altdomherren zählen der Wurzener Ehrenbürger und Superintendent i. R. Horst Schulze, Oberlandeskirchenrat i. R. Dr. Folkert Ihmels und der emeritierte Theologieprofessor Dr. Ernst-Heinz Amberg. Alle Domherren bringen ihren Sachverstand auf den Gebieten Theologie, Bauwesen, Verwaltung, Finanzen, Wirtschaft, Recht und Musik ein. Die ungebrochene Aktivität des Domkapitels zeigt: Das Wurzener Domstift lebt und hat Zukunft.
Domkapitel St. Marien in Wurzen, 2013, in der Mitte der Stiftsherr, Landesbischof Jochen Bohl

29 Archiv des Domstift St. Marien Wurzen, Nr. 1486.1.

30 Siehe die Webseite unter: www. dom-zu-wurzen.de/domkapitel. html [letzter Zugriff: 01.07 . 2015].

\section{Autor}

Pfarrer Alexander

Wieckowski

(2005/06 Vikar in Wurzen)

Großhennersdorf 